\title{
Novel vancomycin resistance gene cluster in Enterococcus faecium ST1486, Belgium, June 2021
}

Basil Britto Xavier ${ }^{1,2}$, Jasmine Coppens ${ }^{3}$, Sien De Koster ${ }^{1,2}$, Sahaya Glingston Rajakani ${ }^{1,2}$, Sam Van Goethem ${ }^{3}$, Samy Mzougui ${ }^{4}$ , Ahalieyah Anantharajah ${ }^{4}$, Christine Lammens ${ }^{1,2}$, Katherine Loens ${ }^{3,5}$, Youri Glupczynski ${ }^{1,2}$, Herman Goossens $\mathbf{s}^{1,2,3,5}$, Veerle Matheeussen 3,5

1. Laboratory of Medical Microbiology, Universiteit Antwerpen, Wilrijk, Belgium

2. Vaccine and Infectious Disease Institute, Universiteit Antwerpen, Wilrijk, Belgium

3. Laboratory of Clinical Microbiology, Antwerp University Hospital, Edegem, Belgium

4. Laboratory of Clinical Microbiology, Cliniques Universitaires Saint-Luc-UCLouvain, Brussels, Belgium

5. Belgian National Reference Centre for Enterococci, Antwerp University Hospital, Edegem, Belgium

Correspondence: Basil Britto Xavier (basilbritto.xavier@uantwerpen.be)

Citation style for this article:

Xavier Basil Britto, Coppens Jasmine, De Koster Sien, Rajakani Sahaya Glingston, Van Goethem Sam, Mzougui Samy, Anantharajah Ahalieyah, Lammens Christine, Loens Katherine, Glupczynski Youri, Goossens Herman, Matheeussen Veerle. Novel vancomycin resistance gene cluster in Enterococcus faecium ST1486, Belgium, June 2021. Euro Surveill. 2021;26(36):pii=2100767. https://doi.org/10.2807/1560-7917.ES.2021.26.36.2100767

We identified a novel van gene cluster in a clinical Enterococcus faecium isolate with vancomycin minimum inhibitory concentration (MIC) of $4 \mu \mathrm{g} /$ $\mathrm{mL}$. The ligase gene, vanP, was part of a van operon cluster of $4,589 \mathrm{bp}$ on a putative novel integrative conjugative element located in a ca98 kb genomic region presumed to be acquired by horizontal gene transfer from Clostridiumscidens and Roseburia sp. 499. Screening for van genes in E. faecium strains with borderline susceptibility to vancomycin is important.

Enterococci are part of the regular intestinal microbiota in humans and animals. In the past two decades, $E$. faecium has rapidly evolved as a worldwide nosocomial pathogen by successfully adapting to the hospital environment $[1,2]$. The World Health Organization (WHO) has described vancomycin-resistant $E$. faecium (VRE) as a high-priority pathogen with urgent need of new treatments [3]. In the present study, we present a case infected with a borderline vancomycinsusceptible E. faecium \#21122516 with a minimum inhibitory concentration (MIC) of $4 \mu \mathrm{g} / \mathrm{mL}$, which grew on a chromogenic VRE-selective agar after a $48 \mathrm{~h}$ incubation but was PCR-negative for all known van genes.

\section{Case report}

In January, 2021, a woman in her 50 s was admitted for cancer surgery to one hospital in Belgium. Her postoperative status in the intensive care unit (ICU) was complicated by the occurrence of ventilatorassociated pneumonia and bacteraemia for which she received multiple courses of broad-spectrum antibiotics (ceftazidime, meropenem, piperacillintazobactam, vancomycin, ciprofloxacin) spanning over a 12-week period. The patient recovered and was transferred after 3 months to a physical rehabilitation unit. Upon admission to the ICU, multiple screening swabs (rectal, upper and lower respiratory tract) taken to detect extended spectrum beta-lactamases, carbapenemase-producing Enterobacteriacae and methicillin-resistant Staphylococcus aureus were negative. However, screening for VRE was not done. After the end of antibiotic therapy (early March 2021), one routine urine specimen yielded a mixed growth of bla ${ }_{\mathrm{NDM}-1}$ carbapenemase-producing Enterobacter cloacae and of $E$. faecium \#21122516, which on BD Phoenix PMIC Panels (New Jersey, United States (US)) was borderline susceptible to vancomycin (MIC $4 \mu \mathrm{g} / \mathrm{mL}$ ) and susceptible to teicoplanin (MIC $<1 \mu \mathrm{g} / \mathrm{mL}$ ). Because this E. faecium strain also grew on a chromogenic selective agar (Chrom ID VRE, bioMérieux, Marcyl'Étoile, France) after $48 \mathrm{~h}$, the isolate was sent as a putative VRE to the Belgian National Reference centre for Enterococci (UZA, Antwerpen, Belgium) for genetic confirmation.

Identification of a novel van gene cluster in a clinical Enterococcus faecium isolate not harbouring any known van genes

Genomic DNA was isolated by use of MagAttract HMW DNA kit (Qiagen, Hilden, Germany) library preparation (Nextera XT), sequenced by MiSeq ( $2 \times 250$ bp, Illumina, San Diego, US). The sequencing data of this study are deposited under the BioProject ID PRJNA741920. Data analysis was done using BacPipe v.2.6.1 [4]. BacPipe results showed the absence of van genes after screening against Resfinder and CARD databases. However, Resfams gave hits to putative glycopeptide resistance genes [5]. The hits containing scaffolds were further analysed using CLC Genomics workbench v.20.1 (clcbio, Aarhus, Denmark) and identified as follows: vanH-vanP-vanX-vanR/walR-vanSG/ 


\section{FIGURE 1}

Genetic organisation of a novel van gene cluster in a putative ICE element, clinical isolate of Enterococcus faecium, Belgium, June 2021

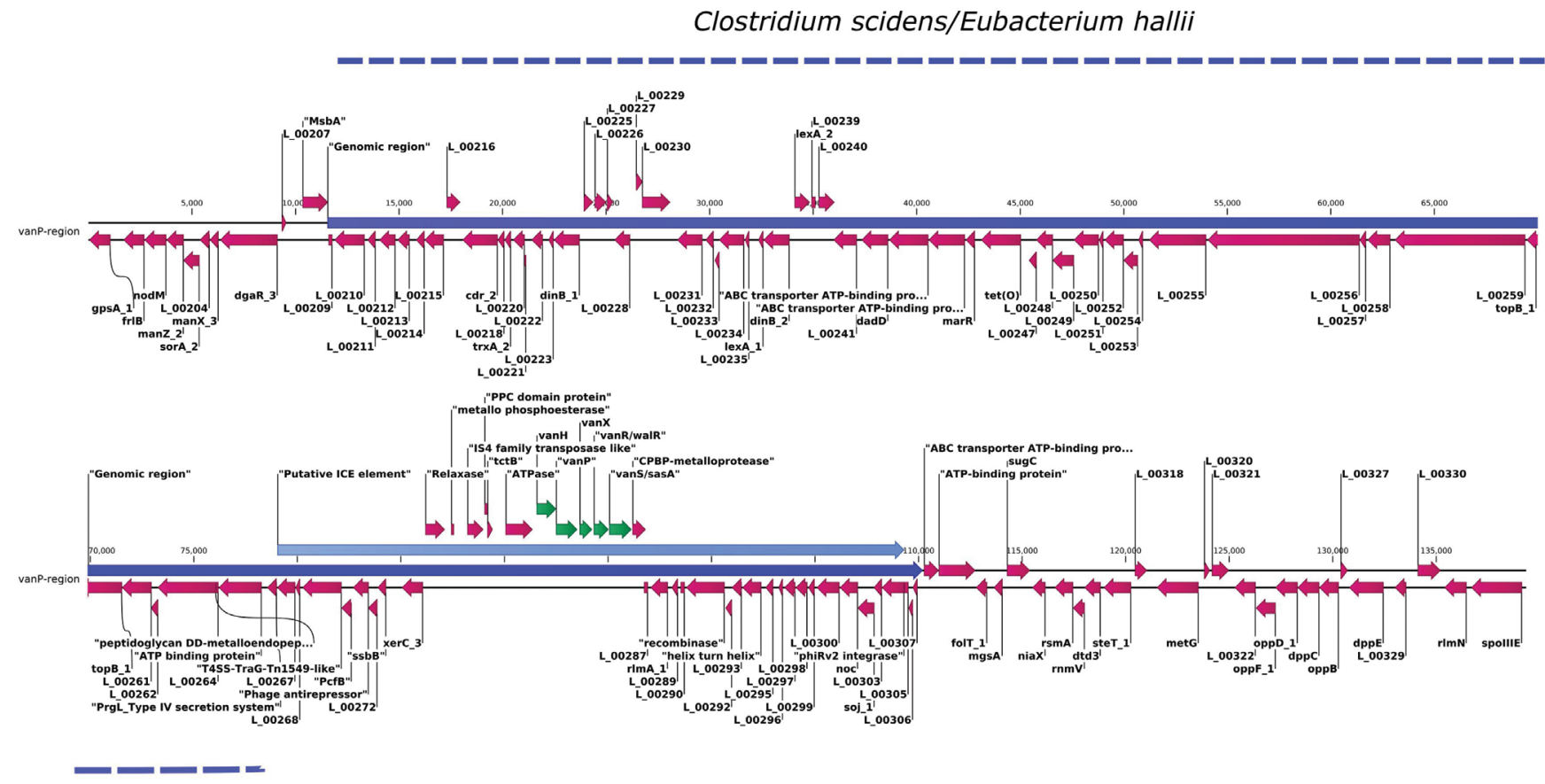

Clostridium scidens/

Eubacterium hallii

ICE: integrative conjugative element.

Green: van gene cluster; light blue: putative ICE element; dotted lines: indicating genomic region shown similarity to Clostridium scidens; dark blue: acquired genomic region.

\section{FIGURE 2}

VanP ligase protein sequence compared parental strain with passaged strains, clinical Enterococcus faecium isolate, Belgium, June 2021

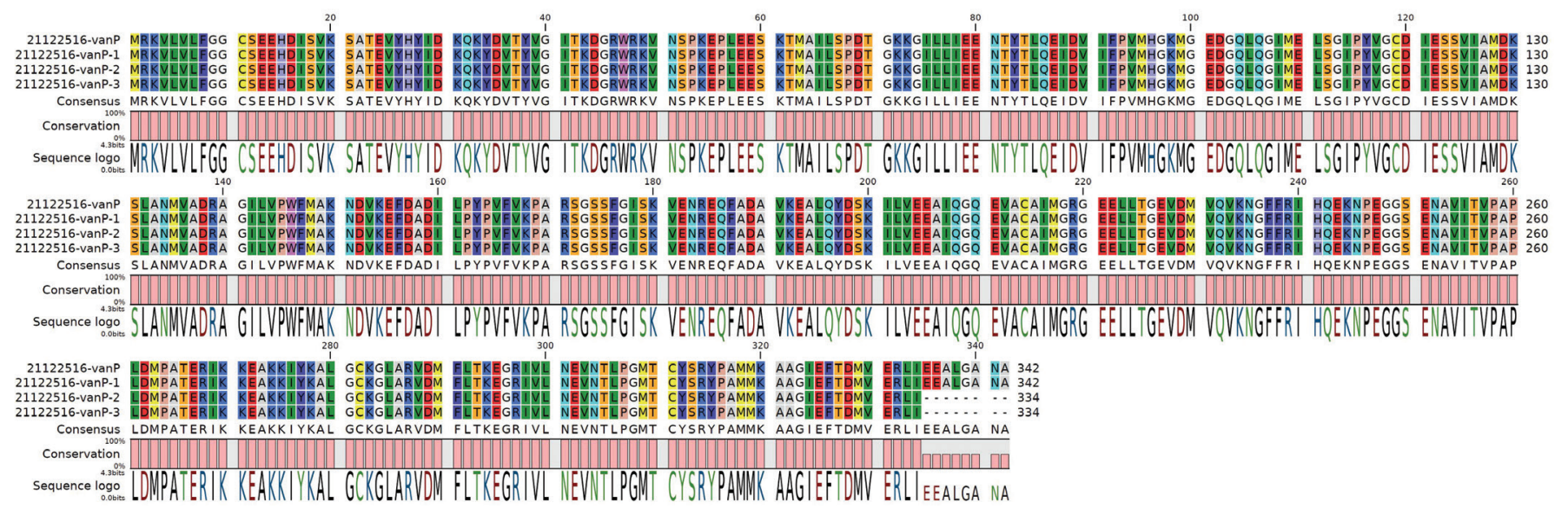


FIGURE 3

Phylogenetic relatedness of D-Ala-D-X ligases from different bacterial species

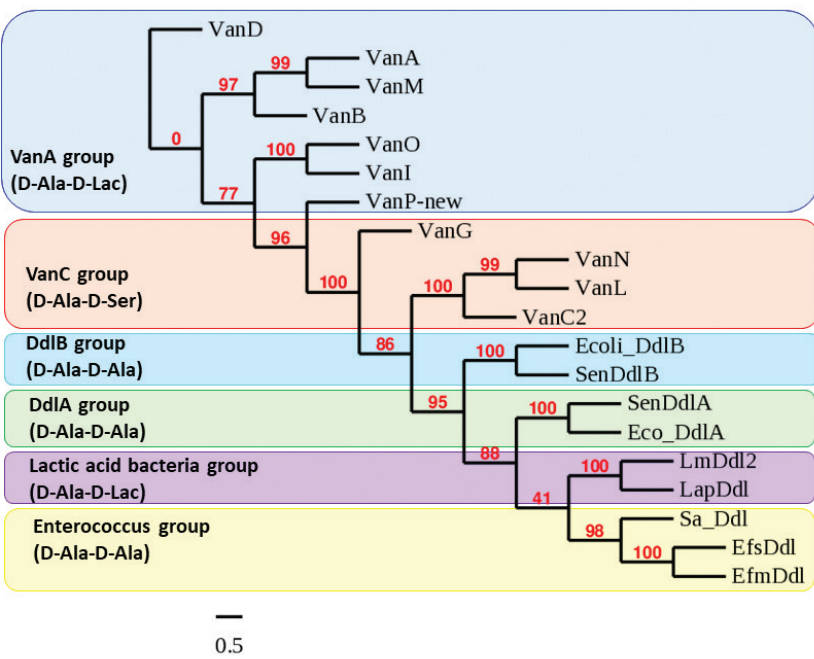

This figure is adapted from Djalal Meziane-Cherif et al. [17]. Bootstrapping analysis of 1,000 replicates was performed using MEGA-X [18] to assess the evolutionary relationship between ligases. Branch lengths represent the number of substitutions per site. Bootstrap values are indicated in the nodes.

sasA with 4,586 bp (Figure 1). BLAST analysis (blastn) of this whole operon gave no hits to any of the organisms in the NCBI non-redundant protein database on 23 July 2021. However, in a BLASTX-based search, the D-alanine-D-alanine ligase domain (WP_075721811) had the highest identity (71.89\%) to Roseburia sp. 499.

Emergence of resistance to vancomycin and teicoplanin following genetic modifications in the ligase gene in serial passaging

By microdilution method, E. faecium \#21122516 (vancomycin (MIC $4 \mu \mathrm{g} / \mathrm{mL}$ ) and teicoplanin (MIC of $\leq 1 \mu \mathrm{g} / \mathrm{mL})$ ) was serially passaged three times in the presence of sub-inhibitory concentrations of vancomycin $(2 \mu \mathrm{g} / \mathrm{mL})$ along with a vancomycinsusceptible (MIC of $1 \mu \mathrm{g} / \mathrm{mL}$ ) control strain E. faecium \#21232802 on a Sensititre Gram-positive EUSTAPF plate (ThermoFisher Scientific, Cleveland, US).

While the vancomycin MIC of the control strain $E$. faecium \#21122516 remained unchanged, resistance emerged for the E. faecium \#21232802 after the first passage (one-fold increase from MIC 4 to $8 \mu \mathrm{g} / \mathrm{mL}$ for vancomycin) while the teicoplanin MIC rose from 0.5 to $2 \mu \mathrm{g} / \mathrm{mL}$. After a second passage, MIC values rose to $>256 \mu \mathrm{g} / \mathrm{mL}$ for vancomycin and $4 \mu \mathrm{g} / \mathrm{mL}$ for teicoplanin. Finally, clones after the third passage showed a marked increase in the MIC to both vancomycin (>256 $\mu \mathrm{g} / \mathrm{ml})$ and teicoplanin $(8 \mu \mathrm{g} / \mathrm{mL})$. The series of three passages was done three times with the same results.

In order to confirm the genetic modifications, whole genome sequencing of in vitro clones from the second and third passages showed a non-synonymous mutation (G to T) in the ligase gene (vanP) (changing Glu 335 to a stop codon) compared with the original strain and with clones from the first passage. This premature stop codon led to a ligase open reading frame $24 \mathrm{nt}$ shorter than that in the parental and once passaged strains (Figure 2). Acquired vancomycin and teicoplanin resistance of $E$. faecium \#21122516 clones was shown to be stable in vitro after five consecutive passages after the appearance of resistance in an antibiotic-free medium.

\section{Acquisition of a large genomic region including a novel van gene cluster on a putative ICE element}

We screened the van gene cluster-containing scaffold for an integrative conjugative element (ICE) using ICEberg 2.0 (http://db-mml.sjtu.edu.cn/ICEberg/) [6]. It predicted the presence of a putative ICE element ca $30 \mathrm{~kb}$ in size, harbouring van genes. This putative ICE element was part of a large genomic region (size ca $98.6 \mathrm{~kb}$ ) acquired by E. faecium \#21122516 and all the components such as type 4 secretion system of Grampositive ICE elements were identified and annotated (data not shown). In order to determine the insertion site, we performed a comparative genome analysis between E. faecium \#21122516 and E. faecium of non-clinical origin NRRL B-2354 (GenBank accession number CPo04063.1). Acquisition had occurred in the insertion site sequence (CAC AAT) between 2,251,669 and $2,252,800 \mathrm{bp}$, in the coding sequence of $m s b A$ for a lipid A export ATP-binding protein. The whole $98.6 \mathrm{~kb}$ region showed similarity to Clostridium scidens (66\% query coverage; $99.26 \%$ identity) and to Eubacterium hallii ( $68 \%$ query coverage: $99.57 \%$ identity). On the other hand, we did not find any similarities to $E$. faecium or any enterococcal genomes when we searched against the NCBI nr database (Figure 1).

\section{Molecular analysis of D-Ala-D-X ligases from Enterococcus faecium \#21122516}

In order to assess the new gene D-Ala-D-X ligase group, we did a phylogenetic analysis of the protein sequences of all known ligase genes taken from GenBank. We further divided the D-Ala-D-X ligases into six major groups based on their phylogenetic relationships. Three were D-Ala-D-Ala ligases, two were D-Ala-D-Lac and one was a D-Ala-D-Ser ligase. The new ligase gene vanP was distantly related to other D-Ala-D-Lac sequences, but clustered within the VanA group (Figure 3).

\section{Genetic characterisation of Enterococcus faecium \#21122516}

The E. faecium \#21122516 strain belongs to sequence type ST1486 of the $\mathrm{CC}_{17}$ complex. In line with the characteristics of a hospital-adapted lineage, this strain harboured multiple resistance genes for trimethoprim $(d f r G)$, tetracycline (tetO), aminoglycoside (aac( $\left.6^{\prime}\right)-l i$, ant (6')-la), aph ( $\left.3^{\prime}\right)-I I I$, macrolide $(\operatorname{msr}(C)$ and virulence factors involved in adhesion to collagen and to adhesive matrix molecules like $\mathrm{acm}$ (a collagen adhesin precursor), sgrA, a cell well-anchored protein that 


\section{FIGURE 4}

Minimum spanning tree comparing core genomic allelic profiles in association with VRE clinical strains isolated in the past 5 years, Belgium 2017-2021

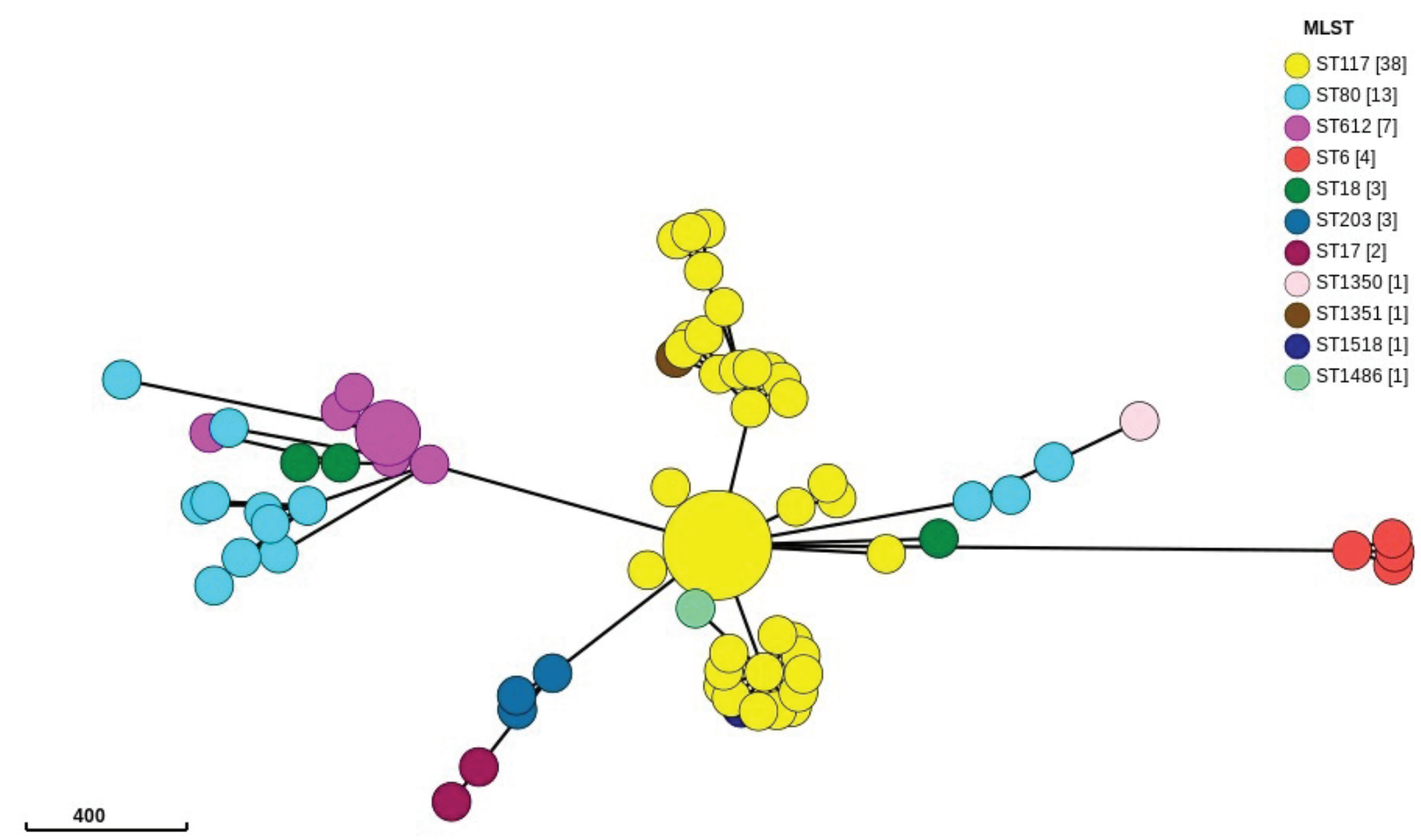

MLST: multilocus sequence typing; VRE: vancomycin-resistant Enterococcus faecium.

Isolates are displayed as circles. Numbers in brackets refer to the number of isolates. The distance between S1486 and ST117 was 79 allelic loci, and both sequence types belonged to the $\mathrm{CC}_{17}$ cluster.

stimulates surface adhesion, ecbA collagen-binding protein $A$ and the esp enterococcal surface protein, which promote biofilm formation. The strain harbours rep14a, rep2 and repUS15 plasmid replicons. We detected eight incomplete phages using the PHASTER online tool [7], indicating that the genome is prone to acquiring new genes.

We looked into the progenitor of E. faecium ST1486 harbouring the van gene cluster. We performed the gene-by-gene approach to determine the core genome [8] multilocus sequence typing (MLST) and whole genome MLST allelic loci distance using chewBBACA [9] and visualised it through Grapetree [10] of the strains sequenced in the past 5 years (Figure 4). A total of 74 E. faecium clinical strains from $\mathrm{CC}_{17}$ complex were randomly selected among the isolates to the reference centre in the period from 2017 to 2021 (Figure 4).

\section{PCR screening for the new vanP gene}

The new vanP gene can be detected by a standard PCR-based screening assay using primers VANP-IF 5' ATGCAGACATTCTTCCTTATCC 3' and VANP-IR 5' GTAACTCCTCTCCTCTTCCC 3 ', and the following cycling conditions: $95^{\circ} \mathrm{C} \times 3 \mathrm{~min}, 25$ cycles of $98^{\circ} \mathrm{C} \times 20 \mathrm{~s}$, $55^{\circ} \mathrm{C} \times 15 \mathrm{~S}, 68^{\circ} \mathrm{C} \times 30 \mathrm{~s}$ and one cycle of $72^{\circ} \mathrm{C} \times 2 \mathrm{~min}$.

\section{Ethical statement}

Approval by an ethics committee was not required for this study because the informed consent from the patient was obtained before investigation. All data were anonymised to protect patient privacy and confidentiality.

\section{Discussion}

We found a newly acquired glycopeptide resistance van gene cluster(vanP) in a clinical strain of $E$. faecium which had occurred as a sporadic isolate in a hospitalised patient following multiple courses of 
antimicrobial therapy. Despite the fact that molecular screening for all known van genes yielded a negative result, we found that resistance to vancomycin and to teicoplanin could be acquired easily and rapidly following exposure to subinhibitory vancomycin concentrations. We could also show that a non-synonymous mutation in the ligase gene created a premature stop codon in the passaged clones, associated with an increase in the vancomycin and teicoplanin MIC. However, actual functional modifications are yet to be explored.

An increase in VRE isolates has been attributed to clonal expansion following gene acquisition [11]. We propose that ST1486 has arisen independently through horizontal gene transfer in hospitals [12]. Roseburia is a Gram-positive intestinal bacterium, and member of Clostridiales, obligate anaerobes that are motile and reported to be difficult to culture and highly susceptible to antimicrobial agents, but homologous vanGlike elements have been reported in Roseburia spp. isolates from the human intestine [13]. As for other types of enterococci, the acquisition and carriage of antibiotic resistance genes is one possible explanation for why $E$. faecium could spread in a hospital setting so quickly [14].

Of the 12 identified van genes, nine have been observed in clinical isolates (vanA, $B, C, D, G, L, M$, N) $[2,15,16]$. It is acknowledged that the predominance of vanA and vanB clusters in major outbreaks is partly due to these clusters being part of successful mobile genetic elements that can disseminate between successful clones. However, selection bias may also contribute to this dominance since in most studies, screening is only performed for the vanA and $\operatorname{van} B$ genes [2].

\section{Conclusion}

We found a novel van gene cluster, van $P$, which was associated with a low level of expression of resistance to vancomycin in a patient clinical isolate of $E$. faecium but caused high-level vancomycin resistance and mediumlevel teicoplanin resistance after exposure to sub-inhibitory concentrations of vancomycin. We hypothesise that this novel van gene cluster was most probably acquired during co-colonisation of Clostridium scidens and Roseburia sp. 499 via horizontal gene transfer under selective pressure by antibiotics. These data suggest that pathogenic enterococci can acquire new vancomycin resistance genes from non-pathogenic species, which can be missed when performing routine screening for vancomycin resistance that is limited to $\operatorname{van} A$ and $\operatorname{van} B$ resistance genes. There is a risk that if they remain unnoticed, some of these strains could proliferate in the hospital setting and pose a threat in the future.

\section{Acknowledgements}

We thank Gert Leten, a senior technician for his technical assistance.

\section{Conflict of interest}

None declared.

\section{Authors' contributions}

This study was conceptualised by BBX. Clinical Isolate and information were collected by SM and AA. Experimental work was done by SDK, SGR, CL, SVG, KL, SM, AA and BBX. Data were analysed and interpreted by BBX, SDK, JC, SGR, CL, SVG, VM, YG and HG. The manuscript was drafted by BBX, SDK, SVG, YG, HG and was reviewed by all authors.

References

1. Bonten MJ, Willems R, Weinstein RA. Vancomycin-resistant enterococci: why are they here, and where do they come from? Lancet Infect Dis. 2001;1(5):314-25. https://doi.org/10.1016/ S1473-3099(01)00145-1 PMID: 11871804

2. Zhou X, Willems RJL, Friedrich AW, Rossen JWA, Bathoorn E. Enterococcus faecium: from microbiological insights to practical recommendations for infection control and diagnostics. Antimicrob Resist Infect Control. 2020;9(1):130. https://doi.org/10.1186/s13756-020-00770-1 PMID: 32778149

3. Tacconelli E, Carrara E, Savoldi A, Harbarth S, Mendelson M, Monnet DL, et al. Discovery, research, and development of new antibiotics: the WHO priority list of antibiotic-resistant bacteria and tuberculosis. Lancet Infect Dis. 2018;18(3):318-27. https:// doi.org/10.1016/S1473-3099(17)30753-3 PMID: 29276051

4. Xavier, B.B., et al., BacPipe: A rapid, user-friendly wholegenome sequencing pipeline for clinical diagnostic bacteriology. iScience. 2020;23(1):100769.

5. Gibson MK, Forsberg KJ, Dantas G. Improved annotation of antibiotic resistance determinants reveals microbial resistomes cluster by ecology. ISME J. 2015;9(1):207-16. https://doi.org/10.1038/ismej.2014.106 PMID: 25003965

6. Liu M, Li X, Xie Y, Bi D, Sun J, Li J, et al. ICEberg 2.0: an updated database of bacterial integrative and conjugative elements. Nucleic Acids Res. 2019;47(D1):D660-5. https://doi. org/10.1093/nar/gky1123 PMID: 30407568

7. Arndt D, Grant JR, Marcu A, Sajed T, Pon A, Liang Y, et al. PHASTER: a better, faster version of the PHAST phage search tool. Nucleic Acids Res. 2016;44(W1):W16-21. https://doi. org/10.1093/nar/gkw387 PMID: 27141966

8. Srinivasan V, Metcalf BJ, Knipe KM, Ouattara M, McGee L, Shewmaker PL, et al. vanG element insertions within a conserved chromosomal site conferring vancomycin resistance to Streptococcus agalactiae and Streptococcus anginosus. MBio. 2014;5(4):e01386-14. https://doi.org/10.1128/ mBio.01386-14 PMID: 25053786

9. Silva M, Machado MP, Silva DN, Rossi M, Moran-Gilad J, Santos $S$, et al. chewBBACA: A complete suite for gene-by-gene schema creation and strain identification. Microb Genom. 2018;4(3). https://doi.org/10.1099/mgen.0.000166 PMID: 29543149

10. Zhou Z, Alikhan NF, Sergeant MJ, Luhmann N, Vaz C, Francisco AP, et al. GrapeTree: visualization of core genomic relationships among 100,000 bacterial pathogens. Genome Res. 2018;28(9):1395-404. https://doi.org/10.1101/ gr.232397.117 PMID: 30049790

11. Ahmed MO, Baptiste KE. Vancomycin-resistant enterococci: a review of antimicrobial resistance mechanisms and perspectives of human and animal health. Microb Drug Resist. 2018;24(5):590-606. https://doi.org/10.1089/mdr.2017.0147 PMID: 29058560

12. Willems RJ, Top J, van Schaik W, Leavis H, Bonten M, Sirén J, et al. Restricted gene flow among hospital subpopulations of Enterococcus faecium. MBio. 2012;3(4):e00151-12. https://doi. org/10.1128/mBio.00151-12 PMID: 22807567

13. Tamanai-Shacoori Z, Smida I, Bousarghin L, Loreal O, Meuric V, Fong SB, et al. Roseburia spp.: a marker of health? Future Microbiol. 2017;12(2):157-70. https://doi.org/10.2217/fmb2016-0130 PMID: 28139139

14. García-Solache M, Rice LB. The Enterococcus: a model of adaptability to its environment. Clin Microbiol Rev. 
2019;32(2):e00058-18. https://doi.org/10.1128/CMR.00058-18 PMID: 30700430

15. Gudeta DD, Moodley A, Bortolaia V, Guardabassi L. vanO, a new glycopeptide resistance operon in environmental

Rhodococcus equi isolates. Antimicrob Agents Chemother. 2014;58(3):1768-70. https://doi.org/10.1128/AAC.01880-13 PMID: 24342631

16. Sivertsen A, Pedersen T, Larssen KW, Bergh K, Rønning TG, Radtke A, et al. A Silenced vanA gene cluster on a transferable plasmid caused an outbreak of vancomycin-variable enterococci. Antimicrob Agents Chemother. 2016;60(7):411927. https://doi.org/10.1128/AAC.00286-16 PMID: 27139479

17. Meziane-Cherif D, Saul FA, Haouz A, Courvalin P. Structural and functional characterization of VanG D-Ala:D-Ser ligase associated with vancomycin resistance in Enterococcus faecalis. J Biol Chem. 2012;287(45):37583-92. https://doi. org/10.1074/jbc.M112.405522 PMID: 22969085

18. Kumar S, Stecher G, Li M, Knyaz C, Tamura K. MEGA X: Molecular evolutionary genetics analysis across computing platforms. Mol Biol Evol. 2018;35(6):1547-9. https://doi. org/10.1093/molbev/msyo96 PMID: 29722887

\section{License, supplementary material and copyright}

This is an open-access article distributed under the terms of the Creative Commons Attribution (CC BY 4.0) Licence. You may share and adapt the material, but must give appropriate credit to the source, provide a link to the licence and indicate if changes were made.

Any supplementary material referenced in the article can be found in the online version.

This article is copyright of the authors or their affiliated institutions, 2021. 